\title{
Asymptotic behavior for a viscoelastic Kirchhoff equation with distributed delay and Balakrishnan-Taylor damping
}

\author{
Abdelbaki Choucha ${ }^{1,2}$ and Salah Boulaaras ${ }^{3,4^{*}}$
}

*Correspondence:

s.boularas@qu.edu.sa

${ }^{3}$ Department of Mathematics,

College of Sciences and Arts, ArRass,

Qassim University, Buraidah, Saudi

Arabia

${ }^{4}$ Laboratory of Fundamental and

Applied Mathematics of Oran

(LMFAO), University of Oran 1, Oran,

31000 Oran, Algeria

Full list of author information is

available at the end of the article

\begin{abstract}
A nonlinear viscoelastic Kirchhoff-type equation with Balakrishnan-Taylor damping and distributed delay is studied. By the energy method we establish the general decay rate under suitable hypothesis.

MSC: 35B40; 35L70; 76Exx; 93D20

Keywords: Kirchhoff equation; Exponential decay; Distributed delay term; Viscoelastic term; Energy method
\end{abstract}

\section{Introduction}

Let $\mathcal{H}=\Omega \times\left(\tau_{1}, \tau_{2}\right) \times(0, \infty)$, in the present work, we consider the following Kirchhoff equation:

$$
\left\{\begin{array}{l}
\left|u_{t}\right|^{p} u_{t t}-\left(\zeta_{0}+\zeta_{1}\|\nabla u\|_{2}^{2}+\sigma\left(\nabla u, \nabla u_{t}\right)_{L^{2}(\Omega)}\right) \Delta u(t)-\Delta u_{t t}(t) \\
\quad+\alpha(t) \int_{0}^{t} h(t-\varrho) \Delta u(\varrho) d \varrho+\beta_{1}\left|u_{t}(t)\right|^{m-2} u_{t}(t) \\
\quad+\int_{\tau_{1}}^{\tau_{2}}\left|\beta_{2}(s)\right|\left|u_{t}(t-s)\right|^{m-2} u_{t}(t-s) d s=0, \\
u(x, 0)=u_{0}(x), \quad u_{t}(x, 0)=u_{1}(x), \quad \text { in } \Omega, \\
u_{t}(x,-t)=f_{0}(x, t), \quad \text { in } \Omega \times\left(0, \tau_{2}\right), \\
u(x, t)=0, \quad \text { in } \partial \Omega \times(0, \infty),
\end{array}\right.
$$

where $\Omega \in \mathbb{R}^{N}$ is a bounded domain with sufficiently smooth boundary $\partial \Omega . \zeta_{0}, \zeta_{1}, \sigma, \beta_{1}$ are positive constants, $p \geq 0$ for $N=1,2$, and $0 \leq p \leq \frac{4}{N-2}$ for $N \geq 3$, and $m \geq 1$ for $N=1,2$, and $1<m \leq \frac{N+2}{N-2}$ for $N \geq 3 . \tau_{1}<\tau_{2}$ are nonnegative constants such that $\beta_{2}:\left[\tau_{1}, \tau_{2}\right] \rightarrow \mathbb{R}$ represents distributive time delay, $h, \alpha$ are positive functions.

Physically, the relationship between the stress and strain history in the beam inspired by Boltzmann theory is called viscoelastic damping term, where the kernel of the term of memory is the function $h$. See [4-6, 9-11, 13-18, 22, 29, 31, 32, 34, 35]. It has been studied by many authors, especially in Kirchhoff's equations (see [8, 10, 19-21, 23-26, 30, 33]).

(c) The Author(s) 2021. This article is licensed under a Creative Commons Attribution 4.0 International License, which permits use, sharing, adaptation, distribution and reproduction in any medium or format, as long as you give appropriate credit to the original author(s) and the source, provide a link to the Creative Commons licence, and indicate if changes were made. The images or other third party material in this article are included in the article's Creative Commons licence, unless indicated otherwise in a credit line to the material. If material is not included in the article's Creative Commons licence and your intended use is not permitted by statutory regulation or exceeds the permitted use, you will need to obtain permission directly from the copyright holder. To view a copy of this licence, visit http://creativecommons.org/licenses/by/4.0/ 
In [2], Balakrishnan and Taylor proposed a new model of damping called the Balakrishnan-Taylor damping, as it relates to the span problem and the plate equation. For more depth, here are some papers that focused on the study of this damping: $[2,3,8,10,16,18$, 20, 27, 35].

The effect of the delay often appears in many applications and practical problems and turns a lot of systems into different problems worth studying. Recently, the stability and the asymptotic behavior of evolution systems with time delay, especially the distributed delay effect, have been studied by many authors. See [7, 10-12, 14, 28].

Based on all of the above, we believe that the combination of these terms of damping (memory term, Balakrishnan-Taylor damping, and the distributed delay) in one particular problem with the addition of $\alpha(t)$ to the term of memory and the distributed delay term $\left(\int_{\tau_{1}}^{\tau_{2}}\left|\beta_{2}(s)\right|\left|u_{t}(t-s)\right|^{m-2} u_{t}(t-s) d s\right)$ constitutes a new problem worthy of study and research, different from the above that we will try to shed light on.

Our paper is divided into several sections: in the next section we lay down the hypotheses, concepts, and lemmas we need, and in the last section we prove our main result.

\section{Preliminaries}

For studying our problem, in this section we will need some materials.

Firstly, we introduce the following hypotheses for $\beta_{2}, h$, and $\alpha$ :

(A1) $h, \alpha: \mathbb{R}_{+} \rightarrow \mathbb{R}_{+}$are nonincreasing $C^{1}$ functions satisfying

$$
\begin{aligned}
& h(t)>0, \quad \alpha(t)>0, \quad l_{0}=\int_{0}^{\infty} h(\varrho) d \varrho<\infty, \\
& \zeta_{0}-2 \alpha(t) \int_{0}^{t} h(\varrho) d \varrho \geq l>0
\end{aligned}
$$

where $l=1-l_{0}$.

(A2) $\exists \vartheta: \mathbb{R}_{+} \rightarrow \mathbb{R}_{+}$is a nonincreasing $C^{1}$ function satisfying

$$
\vartheta(t) h(t)+h^{\prime}(t) \leq 0, \quad t \geq 0 \quad \text { and } \quad \lim _{t \rightarrow \infty} \frac{-\alpha^{\prime}(t)}{\vartheta(t) \alpha(t)}=0
$$

(A3) $\beta_{2}:\left[\tau_{1}, \tau_{2}\right] \rightarrow \mathbb{R}$ is a bounded function satisfying

$$
\int_{\tau_{1}}^{\tau_{2}}\left|\beta_{2}(s)\right| d s<\beta_{1}
$$

Let us introduce

$$
(h \circ \psi)(t):=\int_{\Omega} \int_{0}^{t} h(t-\varrho)|\psi(t)-\psi(\varrho)|^{2} d \varrho d x
$$

and

$$
M(t):=\left(\zeta_{0}+\zeta_{1}\|\nabla u\|_{2}^{2}+\sigma\left(\nabla u(t), \nabla u_{t}(t)\right)_{L^{2}(\Omega)}\right)
$$

Lemma 2.1 (Sobolev-Poincare inequality [1]) Let $2 \leq q<\infty(n=1,2)$ or $2 \leq q<\frac{2 n}{n-2}$ $(n \geq 3)$. Then $\exists c_{*}=c(\Omega, q)>0$ such that

$$
\|u\|_{q} \leq c_{*}\|\nabla u\|_{2}, \quad \forall u \in H_{0}^{1}(\Omega)
$$


As in [28], we take the following new variables:

$$
y(x, \rho, s, t)=u_{t}(x, t-s \rho)
$$

which satisfy

$$
\left\{\begin{array}{l}
s y_{t}(x, \rho, s, t)+y_{\rho}(x, \rho, s, t)=0 \\
y(x, 0, s, t)=u_{t}(x, t)
\end{array}\right.
$$

So, problem (1.1) can be written as

$$
\left\{\begin{array}{l}
\left|u_{t}\right|^{p} u_{t t}-\left(\zeta_{0}+\zeta_{1}\|\nabla u\|_{2}^{2}+\sigma\left(\nabla u, \nabla u_{t}\right)_{L^{2}(\Omega)}\right) \Delta u(t)+\alpha(t) \int_{0}^{t} h(t-\varrho) \Delta u(\varrho) d \varrho \\
\quad-\Delta u_{t t}(t)+\beta_{1}\left|u_{t}(t)\right|^{m-2} u_{t}(t)+\int_{\tau_{1}}^{\tau_{2}}\left|\beta_{2}(s)\right||y(x, 1, s, t)|^{m-2} y(x, 1, s, t) d s=0, \\
s y_{t}(x, \rho, s, t)+y_{\rho}(x, \rho, s, t)=0, \\
u(x, 0)=u_{0}(x), \quad u_{t}(x, 0)=u_{1}(x), \quad \text { in } \Omega, \\
y(x, \rho, s, 0)=f_{0}(x, \rho s), \quad \text { in } \Omega \times(0,1) \times\left(0, \tau_{2}\right), \\
u(x, t)=0, \quad \text { in } \partial \Omega \times(0, \infty),
\end{array}\right.
$$

where

$$
(x, \rho, s, t) \in \Omega \times(0,1) \times\left(\tau_{1}, \tau_{2}\right) \times(0, \infty) .
$$

Now, we give the energy functional.

Lemma 2.2 The energy functional E, defined by

$$
\begin{aligned}
E(t)= & \frac{1}{p+2}\left\|u_{t}\right\|_{p+2}^{p+2}+\frac{1}{2}\left(\zeta_{0}-\alpha(t) \int_{0}^{t} h(\varrho) d \varrho\right)\|\nabla u(t)\|_{2}^{2} \\
& +\frac{1}{2}\left\|\nabla u_{t}(t)\right\|_{2}^{2}+\frac{\zeta_{1}}{4}\|\nabla u(t)\|_{2}^{4}+\frac{\alpha(t)}{2}(h \circ \nabla u)(t) \\
& +\frac{m-1}{m} \int_{0}^{1} \int_{\tau_{1}}^{\tau_{2}} s\left|\beta_{2}(s)\right|\|y(x, \rho, s, t)\|_{m}^{m} d s d \rho,
\end{aligned}
$$

satisfies

$$
\begin{aligned}
E^{\prime}(t) \leq & -\eta_{0}\left\|u_{t}(t)\right\|_{m}^{m}+\frac{\alpha(t)}{2}\left(h^{\prime} \circ \nabla u\right)(t) \\
& -\frac{\alpha^{\prime}(t)}{2}\left(\int_{0}^{t} h(\varrho) d \varrho\right)\|\nabla u(t)\|_{2}^{2}-\frac{\sigma}{4}\left(\frac{d}{d t}\left\{\|\nabla u(t)\|_{2}^{2}\right\}\right)^{2},
\end{aligned}
$$

where $\eta_{0}=\beta_{1}-\int_{\tau_{1}}^{\tau_{2}}\left|\beta_{2}(s)\right| d s>0$.

Proof Taking the inner product of $(2.5)_{1}$ with $u_{t}$, then integrating over $\Omega$, we find

$$
\left(\left|u_{t}\right|^{p} u_{t t}(t), u_{t}(t)\right)_{L^{2}(\Omega)}-\left(M(t) \Delta u(t), u_{t}(t)\right)_{L^{2}(\Omega)}-\left(\Delta u_{t t}(t), u_{t}(t)\right)_{L^{2}(\Omega)}
$$




$$
\begin{aligned}
& +\left(\alpha(t) \int_{0}^{t} h(t-\varrho) \Delta u(\varrho) d \varrho, u_{t}(t)\right)_{L^{2}(\Omega)}+\beta_{1}\left(\left|u_{t}\right|^{m-2} u_{t}, u_{t}\right)_{L^{2}(\Omega)} \\
& +\int_{\tau_{1}}^{\tau_{2}}\left|\beta_{2}(s)\right|\left(|y(x, 1, s, t)|^{m-2} y(x, 1, s, t), u_{t}(t)\right)_{L^{2}(\Omega)} d s=0
\end{aligned}
$$

By computation, integration by parts, and the last condition in (2.5), we get

$$
\begin{aligned}
\left(\left|u_{t}\right|^{p} u_{t t}(t), u_{t}(t)\right)_{L^{2}(\Omega)} & =\int_{\Omega} u_{t}(t) \cdot\left|u_{t}\right|^{p} u_{t t}(t) d x \\
& =\frac{1}{p+2} \frac{d}{d t}\left(\left\|u_{t}(t)\right\|_{p+2}^{p+2}\right), \\
-\left(\Delta u_{t t}(t), u_{t}(t)\right)_{L^{2}(\Omega)} & =\int_{\Omega} \nabla u_{t}(t) \nabla u_{t t}(t) d x \\
& =\frac{1}{2} \frac{d}{d t}\left(\left\|\nabla u_{t}(t)\right\|_{2}^{2}\right) .
\end{aligned}
$$

By integration by parts, we find

$$
\begin{aligned}
-( & \left.M(t) \Delta u(t), u_{t}(t)\right)_{L^{2}(\Omega)} \\
= & -\left(\left(\zeta_{0}+\zeta_{1}\|\nabla u\|_{2}^{2}+\sigma\left(\nabla u(t), \nabla u_{t}(t)\right)_{L^{2}(\Omega)}\right) \Delta u(t), u_{t}(t)\right)_{L^{2}(\Omega)} \\
= & \left(\zeta_{0}+\zeta_{1}\|\nabla u\|_{2}^{2}+\sigma\left(\nabla u(t), \nabla u_{t}(t)\right)_{L^{2}(\Omega)}\right) \int_{\Omega} \nabla u(t) . \nabla u_{t}(t) d x \\
= & \left(\zeta_{0}+\zeta_{1}\|\nabla u\|_{2}^{2}+\sigma\left(\nabla u(t), \nabla u_{t}(t)\right)_{L^{2}(\Omega)}\right) \frac{d}{d t}\left\{\int_{\Omega}|\nabla u(t)|^{2} d x\right\} \\
= & \frac{d}{d t}\left\{\frac{1}{2}\left(\zeta_{0}+\frac{\zeta_{1}}{2}\|\nabla u\|_{2}^{2}\right)\|\nabla u(t)\|_{2}^{2}\right\}+\frac{\sigma}{4} \frac{d}{d t}\left\{\|\nabla u(t)\|_{2}^{2}\right\}^{2},
\end{aligned}
$$

and we have

$$
\begin{aligned}
& \left(\int_{0}^{t} h(t-\varrho) \Delta u(\varrho) d \varrho, u_{t}(t)\right)_{L^{2}(\Omega)} \\
& \quad=\int_{0}^{t} h(t-\varrho)\left(\Delta u(\varrho), u_{t}(t)\right)_{L^{2}(\Omega)} d \varrho \\
& \quad=-\int_{0}^{t} h(t-\varrho)\left[\int_{\Omega} \nabla u(x, \varrho) \nabla u(x, t) d x\right] d \varrho,
\end{aligned}
$$

and

$$
-\nabla u(x, \varrho) . \nabla u(x, t)=\frac{1}{2} \frac{d}{d t}\left\{|\nabla u(x, \varrho)-\nabla u(x, t)(t)|^{2}\right\}-\frac{1}{2} \frac{d}{d t}\left\{|\nabla u(x, t)|^{2}\right\},
$$

then

$$
\begin{aligned}
& -\int_{0}^{t} h(t-\varrho)\left(\nabla u(\varrho), \nabla u_{t}(t)\right)_{L^{2}(\Omega)} d \varrho \\
& \quad=-\int_{0}^{t} h(t-\varrho) \int_{\Omega}\left[\frac{1}{2} \frac{d}{d t}\left\{|\nabla u(x, \varrho)-\nabla u(x, t)|^{2}\right\}\right] d x d s .
\end{aligned}
$$




$$
\begin{aligned}
& -\int_{0}^{t} h(t-\varrho) \int_{\Omega}\left[\frac{1}{2} \frac{d}{d t}\left\{|\nabla u(x, t)|^{2}\right\}\right] d x d \varrho \\
= & \frac{1}{2} \int_{0}^{t} h(t-\varrho)\left[\frac{d}{d t}\left\{\int_{\Omega}|\nabla u(x, t)-\nabla u(x, \varrho)|^{2} d x\right\}\right] d \varrho \\
& -\frac{1}{2} \int_{0}^{t} h(t-\varrho)\left[\frac{d}{d t}\left\{\|\nabla u(x, t)\|_{2}^{2}\right\}\right] d x d \varrho .
\end{aligned}
$$

By (2.1), we get

$$
\begin{aligned}
\frac{1}{2} \int_{0}^{t} h(t-\varrho)\left[\frac{d}{d t}\left\{\int_{\Omega}|\nabla u(x, t)-\nabla u(x, \varrho)|^{2} d x\right\}\right] d \varrho \\
=\frac{1}{2} \frac{d}{d t}\left\{\int_{0}^{t} h(t-\varrho)\left[\int_{\Omega}|\nabla u(x, t)-\nabla u(x, \varrho)|^{2} d x\right]\right\} d \varrho \\
\quad-\frac{1}{2} \int_{0}^{t} h^{\prime}(t-\varrho)\left[\int_{\Omega}|\nabla u(x, t)-\nabla u(x, \varrho)|^{2} d x\right] d \varrho \\
=\frac{1}{2} \frac{d}{d t}(h \circ \nabla u)(t)-\frac{1}{2}\left(h^{\prime} \circ \nabla u\right)(t)
\end{aligned}
$$

and

$$
\begin{aligned}
& -\frac{1}{2} \int_{0}^{t} h(t-\varrho)\left[\frac{d}{d t}\left\{\|\nabla u(t)\|_{2}^{2}\right\}\right] d x d \varrho \\
& \quad=-\frac{1}{2}\left(\int_{0}^{t} h(t-\varrho) d \varrho\right)\left(\frac{d}{d t}\left\{\|\nabla u(t)\|_{2}^{2}\right\}\right) d x \\
& =-\frac{1}{2}\left(\int_{0}^{t} h(\varrho) d \varrho\right)\left(\frac{d}{d t}\left\{\|\nabla u(t)\|_{2}^{2}\right\}\right) d x \\
& =-\frac{1}{2} \frac{d}{d t}\left\{\left(\int_{0}^{t} h(\varrho) d \varrho\right)\|\nabla u(t)\|_{2}^{2}\right\}+\frac{1}{2} h(t)\|\nabla u(t)\|_{2}^{2} .
\end{aligned}
$$

Inserting (2.15) and (2.16) into (2.14) gives

$$
\begin{aligned}
(\alpha(t) & \left.\int_{0}^{t} h(t-\varrho) \Delta u(\varrho) d \varrho, u_{t}(t)\right)_{L^{2}(\Omega)} \\
= & \frac{d}{d t}\left\{\frac{\alpha(t)}{2}(h \circ \nabla u)(t)-\frac{\alpha(t)}{2}\left(\int_{0}^{t} h(\varrho) d \varrho\right)\|\nabla u(t)\|_{2}^{2}\right\} \\
& -\frac{\alpha(t)}{2}\left(h^{\prime} \circ \nabla u\right)(t)+\frac{\alpha(t)}{2} h(t)\|\nabla u(t)\|_{2}^{2} \\
& -\frac{\alpha^{\prime}(t)}{2}(h \circ \nabla u)(t)+\frac{\alpha^{\prime}(t)}{2}\left(\int_{0}^{t} h(\varrho) d \varrho\right)\|\nabla u(t)\|_{2}^{2} .
\end{aligned}
$$

Now, multiplying equation $(2.5)_{2}$ by $-y\left|\beta_{2}(s)\right|$, integrating over $\Omega \times(0,1) \times\left(\tau_{1}, \tau_{2}\right)$, and using $(2.4)_{2}$, we get

$$
\begin{gathered}
\frac{d}{d t} \frac{m-1}{m} \int_{\Omega} \int_{0}^{1} \int_{\tau_{1}}^{\tau_{2}} s\left|\beta_{2}(s)\right| \cdot|y(x, \rho, s, t)|^{m} d s d \rho d x \\
\quad=-(m-1) \int_{\Omega} \int_{0}^{1} \int_{\tau_{1}}^{\tau_{2}}\left|\beta_{2}(s)\right| \cdot|y|^{m-1} y_{\rho} d s d \rho d x
\end{gathered}
$$




$$
\begin{aligned}
= & -\frac{m-1}{m} \int_{\Omega} \int_{0}^{1} \int_{\tau_{1}}^{\tau_{2}}\left|\beta_{2}(\varrho)\right| \frac{d}{d \rho}|y(x, \rho, s, t)|^{m} d s d \rho d x \\
= & \frac{m-1}{m} \int_{\Omega} \int_{\tau_{1}}^{\tau_{2}}\left|\beta_{2}(s)\right|\left(|y(x, 0, s, t)|^{m}-|y(x, 1, s, t)|^{m}\right) d s d x \\
= & \frac{m-1}{m}\left(\int_{\tau_{1}}^{\tau_{2}}\left|\beta_{2}(s)\right| d s\right) \int_{\Omega}\left|u_{t}(t)\right|^{m} d x \\
& -\frac{m-1}{m} \int_{\Omega} \int_{\tau_{1}}^{\tau_{2}}\left|\beta_{2}(s)\right| \cdot|y(x, 1, s, t)|^{m} d s d x \\
= & \frac{m-1}{m}\left(\int_{\tau_{1}}^{\tau_{2}}\left|\beta_{2}(s)\right| d s\right)\left\|u_{t}(t)\right\|_{m}^{m} \\
& -\frac{m-1}{m} \int_{\tau_{1}}^{\tau_{2}}\left|\beta_{2}(s)\right|\|y(x, 1, s, t)\|_{m}^{m} d s .
\end{aligned}
$$

By Young's inequality, we have

$$
\begin{aligned}
& \int_{\tau_{1}}^{\tau_{2}}\left|\beta_{2}(s)\right|\left(|y(x, 1, s, t)|^{m-2} y(x, 1, s, t), u_{t}(t)\right)_{L^{2}(\Omega)} d s \\
& \quad \leq \frac{1}{m}\left(\int_{\tau_{1}}^{\tau_{2}}\left|\beta_{2}(s)\right| d s\right)\left\|u_{t}(t)\right\|_{m}^{m}+\frac{m-1}{m} \int_{\tau_{1}}^{\tau_{2}}\left|\beta_{2}(s)\right|\|y(x, 1, s, t)\|_{m}^{m} d s .
\end{aligned}
$$

By inserting (2.9)-(2.11) and (2.17)-(2.19) into (2.8), we find (2.6) and (2.7).

Hence, by (2.2), we get the function $\mathrm{E}$ is nonincreasing $\forall t \geq t_{1}$. This completes of the proof.

Now we state the local existence of problem (2.5), whose proof can be found in $[23,24]$.

Theorem 2.3 Suppose that (2.1)-(2.3) are satisfied. Then, for any $u_{0}, u_{1} \in H_{0}^{1}(\Omega) \cap L^{2}(\Omega)$, and $f_{0} \in L^{2}\left(\Omega,(0,1),\left(\tau_{1}, \tau_{2}\right)\right)$, there exists a weak solution $u$ of problem $(2.5)$ such that

$$
\begin{aligned}
& u \in C(] 0, T\left[, H_{0}^{1}(\Omega)\right) \cap C^{1}(] 0, T\left[, L^{2}(\Omega)\right) \\
& u_{t} \in C(] 0, T\left[, H_{0}^{1}(\Omega)\right) \cap L^{2}(] 0, T\left[, L^{2}\left(\Omega,(0,1),\left(\tau_{1}, \tau_{2}\right)\right)\right) .
\end{aligned}
$$

\section{General decay}

In this section, we state and prove the asymptotic behavior of system (2.5). For this goal, we set

$$
\Psi(t):=\frac{1}{p+1} \int_{\Omega} u(t)\left|u_{t}\right|^{p} u_{t}(t) d x+\frac{\sigma}{4}\|\nabla u(t)\|_{2}^{4}+\int_{\Omega} \nabla u(t) \nabla u_{t}(t) d x
$$

and

$$
\Phi(t):=\int_{\Omega}\left(\Delta u_{t}-\frac{1}{p+1}\left|u_{t}\right|^{p} u_{t}\right) \int_{0}^{t} h(t-\varrho)(u(t)-u(\varrho)) d \varrho d x
$$

and

$$
\Theta(t):=\int_{0}^{1} \int_{\tau_{1}}^{\tau_{2}} s e^{-\rho s}\left|\beta_{2}(s)\right| \cdot\|y(x, \rho, s, t)\|_{m}^{m} d s d \rho .
$$


First, since the function $h$ is positive and continuous, for all $t_{0}>0$, we have

$$
\int_{0}^{t} h(\varrho) d \varrho \geq \int_{0}^{t_{0}} h(\varrho) d \varrho:=h_{0}, \quad \forall t \geq t_{0} .
$$

Lemma 3.1 The functional $\Psi(t)$ defined in (3.1) satisfies, for any $\varepsilon>0$,

$$
\begin{aligned}
\Psi^{\prime}(t) \leq & \frac{1}{p+1}\left\|u_{t}\right\|_{p+2}^{p+2}-\left(l-\varepsilon\left(c_{1}+c_{2}\right)\right)\|\nabla u\|_{2}^{2}-\zeta_{1}\|\nabla u\|_{2}^{4}+\frac{\alpha(t)}{4}(h \circ \nabla u)(t) \\
& +\left\|\nabla u_{t}\right\|_{2}^{2}+c(\varepsilon)\left(\left\|u_{t}\right\|_{m}^{m}+\int_{\tau_{1}}^{\tau_{2}}\left|\beta_{2}(s)\right|\|y(x, 1, s, t)\|_{m}^{m} d s\right)
\end{aligned}
$$

Proof A differentiation of (3.1) and using (2.5) 1 give

$$
\begin{aligned}
& \Psi^{\prime}(t)=\frac{1}{p+1}\left\|u_{t}\right\|_{p+2}^{p+2}+\int_{\Omega}\left|u_{t}\right|^{p} u_{t t} u d x+\sigma\|\nabla u\|_{2}^{2} \int_{\Omega} \nabla u_{t} \nabla u d x \\
& +\int_{\Omega} \nabla u(t) \nabla u_{t t}(t) d x+\left\|\nabla u_{t}\right\|_{2}^{2} \\
& =\frac{1}{p+1}\left\|u_{t}\right\|_{p+2}^{p+2}-\zeta_{0}\|\nabla u\|_{2}^{2}-\zeta_{1}\|\nabla u\|_{2}^{4} \underbrace{-\beta_{1} \int_{\Omega}\left|u_{t}\right|^{m-2} u_{t} u d x}_{J_{1}} \\
& +\underbrace{\alpha(t) \int_{\Omega} \nabla u(t) \int_{0}^{t} h(t-\varrho) \nabla u(\varrho) d \varrho d x}_{J_{2}}+\left\|\nabla u_{t}\right\|_{2}^{2} \\
& -\underbrace{\int_{\Omega} \int_{\tau_{1}}^{\tau_{2}}\left|\beta_{2}(s)\right||y(x, 1, s, t)|^{m-2} y(x, 1, s, t) . u d s d x}_{J_{3}}
\end{aligned}
$$

We estimate the last three terms of the RHS of (3.5). Applying Hölder's, Sobolev-Poincare, and Young's inequalities, (2.1) and (2.6), we find

$$
\begin{aligned}
J_{1} & \leq \varepsilon \beta_{1}^{m}\|u\|_{m}^{m}+c(\varepsilon)\left\|u_{t}\right\|_{m}^{m} \\
& \leq \varepsilon \beta_{1}^{m} c_{p}^{m}\|\nabla u\|_{2}^{m}+c(\varepsilon)\left\|u_{t}\right\|_{m}^{m} \\
& \leq \varepsilon \beta_{1}^{m} c_{p}^{m}\left(\frac{E(0)}{l}\right)^{(m-2) / 2}\|\nabla u\|_{2}^{2}+c(\varepsilon)\left\|u_{t}\right\|_{m}^{m} \\
& \leq \varepsilon c_{1}\|\nabla u\|_{2}^{2}+c(\varepsilon)\left\|u_{t}\right\|_{m}^{m}
\end{aligned}
$$

and

$$
\begin{aligned}
J_{2} & \leq 2 \alpha(t)\left(\int_{0}^{t} h(\varrho) d \varrho\right)\|\nabla u\|_{2}^{2}+\frac{\alpha(t)}{4}(h \circ \nabla u)(t) \\
& \leq\left(\zeta_{0}-l\right)\|\nabla u\|_{2}^{2}+\frac{\alpha(t)}{4}(h \circ \nabla u)(t) .
\end{aligned}
$$

Similar to $J_{1}$, we have

$$
J_{3} \leq \varepsilon c_{2}\|\nabla u\|_{2}^{2}+c(\varepsilon) \int_{\tau_{1}}^{\tau_{2}}\left|\beta_{2}(s)\right| \cdot\|y(x, 1, s, t)\|_{m}^{m} d s .
$$


Combining (3.6)-(3.8) and (3.5), we get

$$
\begin{aligned}
\Psi^{\prime}(t) \leq & \frac{1}{p+1}\left\|u_{t}\right\|_{p+2}^{p+2}-\left(l-\varepsilon\left(c_{1}+c_{2}\right)\right)\|\nabla u\|_{2}^{2}-\zeta_{1}\|\nabla u\|_{2}^{4}+\left\|\nabla u_{t}\right\|_{2}^{2} \\
& +\frac{\alpha(t)}{4}(h \circ \nabla u)(t)+c(\varepsilon)\left(\left\|u_{t}\right\|_{m}^{m}+\int_{\tau_{1}}^{\tau_{2}}\left|\beta_{2}(s)\right|\|y(x, 1, s, t)\|_{m}^{m} d s\right) .
\end{aligned}
$$

Lemma 3.2 The functional $\Phi(t)$ defined in (3.2) satisfies, for any $\delta>0$,

$$
\begin{aligned}
\Phi^{\prime}(t) \leq & -\frac{1}{p+1}\left(\int_{0}^{t} h(\varrho) d \varrho\right)\left\|u_{t}\right\|_{p+2}^{p+2}+\delta\left(\zeta_{0}+2 h_{0}^{2} \alpha(t)\right)\|\nabla u\|_{2}^{2} \\
& +\zeta_{1} \delta\|\nabla u\|_{2}^{4}+\delta \frac{\sigma E(0)}{l}\left(\frac{1}{2} \frac{d}{d t}\|\nabla u\|_{2}^{2}\right)^{2} \\
& +\left(c(\delta)+\left(2 \delta+\frac{1}{4 \delta}\right) c \alpha(t)\right)(h \circ \nabla u)(t) \\
& +c(\delta)\left(\left\|u_{t}\right\|_{m}^{m}+\int_{\tau_{1}}^{\tau_{2}}\left|\beta_{2}(\varrho)\right|\|y(x, 1, s, t)\|_{m}^{m} d s\right) \\
& +\left(\delta_{1}\left(1+c(E(0))^{p}\right)-\int_{0}^{t} h(\varrho) d \varrho\right)\left\|\nabla u_{t}\right\|_{2}^{2} \\
& -\left(\frac{h(0) c_{p}^{2}}{4 \delta_{1}}+c\left(\delta_{1}\right)\right)\left(h^{\prime} \circ \nabla u\right)(t) .
\end{aligned}
$$

Proof A differentiation of (3.2) and using (2.5) ${ }_{1}$ give

$$
\begin{aligned}
& \Phi^{\prime}(t)=\int_{\Omega}\left(\Delta u_{t t}-u_{t t}\left|u_{t}\right|^{p}\right) \int_{0}^{t} h(t-\varrho)(u(t)-u(\varrho)) d \varrho d x \\
& +\int_{\Omega}\left(\Delta u_{t}-\frac{1}{p+1}\left|u_{t}\right|^{p} u_{t}\right) \int_{0}^{t} h^{\prime}(t-\varrho)(u(t)-u(\varrho)) d \varrho d x \\
& -\frac{1}{p+1}\left(\int_{0}^{t} h(\varrho) d \varrho\right)\left\|u_{t}\right\|_{p+2}^{p+2}-\left(\int_{0}^{t} h(\varrho) d \varrho\right)\left\|\nabla u_{t}\right\|_{2}^{2} \\
& =\underbrace{\left(\zeta_{0}+\zeta_{1}\|\nabla u\|_{2}^{2}\right) \int_{\Omega} \nabla u \int_{0}^{t} h(t-\varrho)(\nabla u(t)-\nabla u(\varrho)) d \varrho d x}_{J_{1}} \\
& \underbrace{+\sigma \int_{\Omega} \nabla u \nabla u_{t} d x \cdot \int_{\Omega} \nabla u \int_{0}^{t} h(t-\varrho)(\nabla u(t)-\nabla u(\varrho)) d \varrho d x}_{J_{2}} \\
& \underbrace{-\alpha(t) \int_{\Omega}\left(\int_{0}^{t} h(t-\varrho) \nabla u(\varrho) d \varrho\right) \cdot\left(\int_{0}^{t} h(t-\varrho)(\nabla u(t)-\nabla u(\varrho)) d \varrho\right) d x}_{J_{3}} \\
& \underbrace{-\beta_{1} \int_{\Omega}\left|u_{t}\right|^{m-2} u_{t}\left(\int_{0}^{t} h(t-\varrho)(u(t)-u(\varrho)) d \varrho\right) d x}_{J_{4}} \\
& -\int_{\Omega} \int_{\tau_{1}}^{\tau_{2}}\left|\beta_{2}(s)\right||y(x, 1, s, t)|^{m-2} y(x, 1, s, t)
\end{aligned}
$$




$$
\begin{aligned}
& \underbrace{\times\left(\int_{0}^{t} h(t-\varrho)(u(t)-u(\varrho)) d \varrho\right) d s d x}_{J_{5}} \\
& \underbrace{-\frac{1}{p+1} \int_{\Omega}\left|u_{t}\right|^{p} u_{t} \int_{0}^{t} h^{\prime}(t-\varrho)(u(t)-u(\varrho)) d \varrho d x}_{J_{6}} \\
& \underbrace{-\int_{\Omega} \nabla u_{t} \int_{0}^{t} h^{\prime}(t-\varrho)(\nabla u(t)-\nabla u(\varrho)) d \varrho d x}_{J_{7}} \\
& -\frac{1}{p+1}\left(\int_{0}^{t} h(\varrho) d \varrho\right)\left\|u_{t}\right\|_{p+2}^{p+2}-\left(\int_{0}^{t} h(\varrho) d \varrho\right)\left\|\nabla u_{t}\right\|_{2}^{2} .
\end{aligned}
$$

By estimating the terms $J_{i}, i=1, \ldots, 7$, of the RHS of (3.10), exploiting Hölder's, SobolevPoincare, and Young's inequalities, (2.1) and (2.6), we find

$$
\begin{aligned}
\left|J_{1}\right| & \leq\left(\zeta_{0}+\zeta_{1}\|\nabla u\|_{2}^{2}\right)\left(\delta\|\nabla u\|_{2}^{2}+\frac{c}{4 \delta}(h \circ \nabla u)(t)\right) \\
& \leq \delta \zeta_{0}\|\nabla u\|_{2}^{2}+\delta \zeta_{1}\|\nabla u\|_{2}^{4}+\left(\frac{\zeta_{0} c}{4 \delta}+\frac{\zeta_{1} c E(0)}{4 l \delta}\right)(h \circ \nabla u)(t)
\end{aligned}
$$

and

$$
\begin{aligned}
J_{2} \leq & \delta \sigma\left(\int_{\Omega} \nabla u \nabla u_{t} d x\right)^{2}\|\nabla u\|_{2}^{2}+\frac{\sigma c}{4 \delta}(h \circ \nabla u)(t) \\
\leq & \delta \frac{\sigma E(0)}{l}\left(\frac{1}{2} \frac{d}{d t}\|\nabla u\|_{2}^{2}\right)^{2}+\frac{\sigma c}{4 \delta}(h \circ \nabla u)(t), \\
\left|J_{3}\right| \leq & \delta \alpha(t) \int_{\Omega}\left(\int_{0}^{t} h(t-\varrho)(|\nabla u(t)-\nabla u(\varrho)|-\nabla|u(t)|) d \varrho\right)^{2} d x \\
& +\frac{1}{4 \delta} \alpha(t) \int_{\Omega}\left(\int_{0}^{t} h(t-\varrho)(\nabla u(t)-\nabla u(\varrho)) d \varrho\right)^{2} d x \\
\leq & 2 \delta h_{0}^{2} \alpha(t)\|\nabla u\|_{2}^{2}+\left(2 \delta+\frac{1}{4 \delta}\right) c \alpha(t)(h \circ \nabla u)(t), \\
J_{4} \mid \leq & c(\delta)\left\|u_{t}\right\|_{m}^{m}+\delta \beta_{1}^{m} \int_{\Omega}\left(\int_{0}^{t} h(t-\varrho)(u(t)-u(\varrho)) d \varrho\right)^{m} d x \\
\leq & c(\delta)\left\|u_{t}\right\|_{m}^{m}+\delta \beta_{1}^{m} c_{p}^{m} \int_{0}^{t} h(t-\varrho)\|\nabla u(t)-\nabla u(\varrho)\|_{2}^{m} d \varrho \\
\leq & c(\delta)\left\|u_{t}\right\|_{m}^{m}+\delta\left(\beta_{1}^{m} c_{p}^{m}\left(\frac{E(0)}{l}\right)^{(m-2) / 2}\right)(h \circ \nabla u)(t) \\
\leq & c(\delta)\left\|u_{t}\right\|_{m}^{m}+\delta c_{3}(h \circ \nabla u)(t) .
\end{aligned}
$$

Similarly, we have

$$
\left|J_{5}\right| \leq c(\delta)\|y(x, 1, s, t)\|_{m}^{m}+\delta c_{4}(h \circ \nabla u)(t)
$$


By exploiting the Sobolev embedding, we have

$$
\begin{aligned}
\left|J_{6}\right| & \leq \frac{1}{p+1}\left(\delta_{1}\left\|u_{t}\right\|_{2(p+1)}^{2(p+1)}+\frac{c}{\delta_{1}} \int_{\Omega} \int_{0}^{t}\left(-h^{\prime}(t-\varrho)\right)|u(t)-u(\varrho)|^{2} d \varrho d x\right) \\
& \leq c \delta_{1}(E(0))^{p}\left\|\nabla u_{t}\right\|_{2}^{2}-c\left(\delta_{1}\right)\left(h^{\prime} \circ \nabla u\right)(t)
\end{aligned}
$$

and

$$
\left|J_{7}\right| \leq \delta_{1}\left\|\nabla u_{t}\right\|_{2}^{2}-\frac{h(0)}{4 \delta_{1}}\left(h^{\prime} \circ \nabla u\right)(t) .
$$

According to (3.11)-(3.17) and (3.10), we get (3.9).

Lemma 3.3 The functional $\Theta(t)$ defined in (3.3) satisfies

$$
\begin{aligned}
\Theta^{\prime}(t) \leq & -\eta_{1} \int_{0}^{1} \int_{\tau_{1}}^{\tau_{2}} s\left|\beta_{2}(s)\right| \cdot\|y(x, \rho, s, t)\|_{m}^{m} d s d \rho \\
& -\eta_{1} \int_{\tau_{1}}^{\tau_{2}}\left|\beta_{2}(s)\right| \cdot\|y(x, 1, s, t)\|_{m}^{m} d s+\beta_{1}\left\|u_{t}(t)\right\|_{m}^{m} .
\end{aligned}
$$

Proof Differentiating $\Theta(t)$ and using $(2.5)_{2}$ give

$$
\begin{aligned}
\Theta^{\prime}(t)= & -m \int_{\Omega} \int_{0}^{1} \int_{\tau_{1}}^{\tau_{2}} e^{-s \rho}\left|\beta_{2}(s)\right| \cdot|y|^{m-1} y_{\rho}(x, \rho, s, t) d s d \rho d x \\
= & -\int_{\Omega} \int_{0}^{1} \int_{\tau_{1}}^{\tau_{2}} s e^{-s \rho}\left|\beta_{2}(s)\right| \cdot|y(x, \rho, s, t)|^{m} d s d \rho d x \\
& -\int_{\Omega} \int_{\tau_{1}}^{\tau_{2}}\left|\beta_{2}(s)\right|\left[e^{-s}|y(x, 1, s, t)|^{m}-|y(x, 0, s, t)|^{m}\right] d s d x
\end{aligned}
$$

Applying $y(x, 0, s, t)=u_{t}(x, t)$ and $e^{-s} \leq e^{-s \rho} \leq 1$ for any $0<\rho<1$ and setting $\eta_{1}=e^{-\tau_{2}}$, we obtain

$$
\begin{aligned}
\Theta^{\prime}(t) \leq & -\eta_{1} \int_{\Omega} \int_{0}^{1} \int_{\tau_{1}}^{\tau_{2}} s\left|\beta_{2}(s)\right| \cdot|y(x, \rho, s, t)|^{m} d s d \rho d x \\
& -\eta_{1} \int_{\Omega} \int_{\tau_{1}}^{\tau_{2}}\left|\beta_{2}(s)\right||y(x, 1, s, t)|^{m} d s d x+\int_{\tau_{1}}^{\tau_{2}}\left|\beta_{2}(s)\right| d s \int_{\Omega}\left|u_{t}\right|^{m}(t) d x
\end{aligned}
$$

using (2.3), we find (3.18).

Now, we introduce the functional

$$
\mathcal{G}(t):=E(t)+\varepsilon_{1} \alpha(t) \Psi(t)+\varepsilon_{2} \alpha(t) \Phi(t)+\varepsilon_{3} \alpha(t) \Theta(t)
$$

for some positive constants $\varepsilon_{i}, i=1,2,3$, to be determined.

Lemma 3.4 There exist $\mu_{1}, \mu_{2}>0$ such that

$$
\mu_{1} E(t) \leq \mathcal{G}(t) \leq \mu_{2} E(t) .
$$


Proof From (3.1), by using Hölder's inequality (for $q_{1}=\frac{p+2}{p+1}, q_{2}=p+2$ ), Young's, and Poincare inequalities (for $\kappa>0$ ), and $\left\|u_{t}\right\|_{p+2}^{p} \leq[(p+2) E(0)]^{\frac{p}{(p+2)}}$, we find

$$
\begin{aligned}
\Psi(t) \leq & \frac{1}{p+1}\left\|u_{t}(t)\right\|_{p+2}^{p+1}\|u(t)\|_{p+2}+\frac{1}{2}\left(\left\|\nabla u_{t}(t)\right\|_{2}^{2}+\|\nabla u(t)\|_{2}^{2}\right) \\
\leq & \frac{\kappa}{2(p+1)^{2}}\left\|u_{t}(t)\right\|_{p+2}^{2(p+1)}+\frac{1}{2 \kappa}\|u(t)\|_{p+2}^{2} \\
& +\frac{1}{2}\left(\left\|\nabla u_{t}(t)\right\|_{2}^{2}+\|\nabla u(t)\|_{2}^{2}\right) \\
\leq & \frac{\kappa}{2(p+1)^{2}}\left\|u_{t}(t)\right\|_{p+2}^{p}\left\|u_{t}(t)\right\|_{p+2}^{p+2}+\frac{1}{2 \kappa}\|u(t)\|_{p+2}^{2} \\
& +\frac{1}{2}\left(\left\|\nabla u_{t}(t)\right\|_{2}^{2}+\|\nabla u(t)\|_{2}^{2}\right) \\
\leq & \frac{\kappa[(p+2) E(0)]^{\frac{p}{(p+2)}}}{2(p+1)^{2}}\left\|u_{t}(t)\right\|_{p+2}^{p+2}+c(\kappa)\|\nabla u(t)\|_{2}^{2}+\frac{1}{2}\left\|\nabla u_{t}(t)\right\|_{2}^{2},
\end{aligned}
$$

where $c(\kappa)=\left(\frac{C_{0}}{2 \kappa}+\frac{1}{2}\right)$.

According to (3.21) and from (3.2)-(3.3), we get

$$
\begin{aligned}
|\mathcal{G}(t)-E(t)| \leq & \varepsilon_{1}|\alpha(t)|\left(\frac{\kappa[(p+2) E(0)] \frac{p}{(p+2)}}{2(p+1)^{2}}\left\|u_{t}(t)\right\|_{p+2}^{p+2}+c(\kappa)\|\nabla u(t)\|_{2}^{2}\right) \\
& +\left(\varepsilon_{1}+\varepsilon_{2}\right) \frac{|\alpha(t)|}{2}\left\|\nabla u_{t}(t)\right\|_{2}^{2}+\varepsilon_{1} \sigma \frac{|\alpha(t)|}{4}\|\nabla u(t)\|_{2}^{4} \\
& +\varepsilon_{2} \frac{|\alpha(t)|}{2(p+1)}\left\|u_{t}(t)\right\|_{2(p+1)}^{2(p+1)}+\varepsilon_{2} \frac{|\alpha(t)|\left(\zeta_{0}-l\right) c(p)}{2}(h \circ \nabla u)(t) \\
& +\varepsilon_{3}|\alpha(t)| \int_{0}^{1} \int_{\tau_{1}}^{\tau_{2}} s e^{-\rho s}\left|\beta_{2}(s)\right| .\|y(x, \rho, s, t)\|_{m}^{m} d s d \rho,
\end{aligned}
$$

where $c(p)=\left(\frac{c_{p}}{p+1}+1\right)$.

Using the fact that $0<\alpha(t) \leq \alpha(0)$ and $e^{-\rho s}<1$, we find

$$
\begin{aligned}
|\mathcal{G}(t)-E(t)| \leq & \varepsilon_{1} \alpha(0)\left(\frac{\kappa[(p+2) E(0)]^{\frac{p}{(p+2)}}}{2(p+1)^{2}}\left\|u_{t}(t)\right\|_{p+2}^{p+2}+c(\kappa)\|\nabla u(t)\|_{2}^{2}\right) \\
& +\left(\varepsilon_{1}+\varepsilon_{2}\right) \frac{|\alpha(0)|}{2}\left\|\nabla u_{t}(t)\right\|_{2}^{2}+\varepsilon_{1} \sigma \frac{|\alpha(0)|}{4}\|\nabla u(t)\|_{2}^{4} \\
& +\varepsilon_{2} \frac{|\alpha(0)| c(E(0))^{p}}{2(p+1)}\left\|\nabla u_{t}(t)\right\|_{2}^{2}+\varepsilon_{2} \frac{|\alpha(0)|\left(\zeta_{0}-l\right) c(p)}{2}(h \circ \nabla u)(t) \\
& +\varepsilon_{3} \alpha(0) \int_{0}^{1} \int_{\tau_{1}}^{\tau_{2}} s e^{-\rho s}\left|\beta_{2}(s)\right| \cdot\|y(x, \rho, s, t)\|_{m}^{m} d s d \rho \\
\leq & C\left(\varepsilon_{1}, \varepsilon_{2}, \varepsilon_{3}, \kappa\right) E(t) .
\end{aligned}
$$

We pick $\kappa=1$ and choose $\varepsilon_{1}, \varepsilon_{2}$, and $\varepsilon_{3}$ sufficiently small, then (3.20) follows from (3.23).

Lemma 3.5 There exist $k_{7}, k_{8}, t_{0}>0$ satisfying

$$
\mathcal{G}^{\prime}(t) \leq-k_{7} \alpha(t) E(t)+k_{8} \alpha(t)(h \circ \nabla u)(t), \quad t>t_{0} .
$$


Proof A differentiation of (3.19), using (2.7), Lemmas 3.1, 3.2, and 3.3 lead to

$$
\begin{aligned}
\mathcal{G}^{\prime}(t):= & E^{\prime}(t)+\varepsilon_{1} \alpha^{\prime}(t) \Psi(t)+\varepsilon_{2} \alpha^{\prime}(t) \Phi(t)+\varepsilon_{3} \alpha^{\prime}(t) \Theta(t) \\
& +\varepsilon_{1} \alpha(t) \Psi^{\prime}(t)+\varepsilon_{2} \alpha(t) \Phi^{\prime}(t)+\varepsilon_{3} \alpha(t) \Theta^{\prime}(t) .
\end{aligned}
$$

By using the fact that $e^{-\rho s}<1$, Young's and Sobolev-Poincare inequalities, we find

$$
\begin{aligned}
\alpha^{\prime}(t) & \left(\varepsilon_{1} \Psi(t)+\varepsilon_{2} \Phi(t)+\varepsilon_{3} \Theta(t)\right) \\
\leq & -\alpha^{\prime}(t)\left\{\varepsilon_{1} C_{1}\left\|u_{t}\right\|_{p+2}^{p+2}+\varepsilon_{1} c(\kappa)\|\nabla u\|_{2}^{2}+\frac{1}{2}\left(\varepsilon_{1}+\varepsilon_{2} C_{2}\right)\left\|\nabla u_{t}\right\|_{2}^{2}\right. \\
& \left.\left.+\varepsilon_{2} C_{3} h \circ \nabla u\right)(t)+\varepsilon_{3} \int_{0}^{1} \int_{\tau_{1}}^{\tau_{2}} s\left|\beta_{2}(s)\right| \cdot\|y(x, \rho, s, t)\|_{m}^{m} d s d \rho\right\},
\end{aligned}
$$

where $C_{1}=\frac{\kappa[(p+2) E(0)]^{p /(p+2)}}{2(p+1)^{2}}>0, C_{2}=1+\frac{c(E(0))^{p}}{p+1}>0$, and $C_{3}=\frac{\left(\zeta_{0}-l\right) c(p)}{2}>0$.

Hence, by using (2.7), Lemmas 3.1, 3.2, 3.3, and (3.26), we get

$$
\begin{aligned}
& \mathcal{G}^{\prime}(t) \leq \alpha(t)\left\{\frac{1}{p+1}\left(\varepsilon_{1}-\varepsilon_{2} h_{0}\right)-\varepsilon_{1} \frac{\alpha^{\prime}(t)}{\alpha(t)} C_{1}\right\}\left\|u_{t}\right\|_{p+2}^{p+2} \\
& +\alpha(t)\left\{\varepsilon_{2} \delta\left(\zeta_{0}+2 h_{0}^{2} \alpha(t)\right)-\varepsilon_{1}\left(l-\varepsilon\left(c_{1}+c_{2}\right)\right)\right. \\
& \left.-\frac{\alpha^{\prime}(t)}{2 \alpha(t)}\left(\int_{0}^{t} h(\varrho) d \varrho\right)-\varepsilon_{1} \frac{\alpha^{\prime}(t) c(\kappa)}{\alpha(t)}\right\}\|\nabla u\|_{2}^{2} \\
& +\alpha(t)\left\{\varepsilon_{1}+\varepsilon_{2}\left[\delta_{1}\left(1+c(E(0))^{p} \alpha(t)\right)-h_{0}\right]-\frac{\alpha^{\prime}(t)}{2 \alpha(t)}\left(\varepsilon_{1}+\varepsilon_{2} C_{2}\right)\right\}\left\|\nabla u_{t}\right\|_{2}^{2} \\
& +\alpha(t)\left\{\varepsilon_{2} \zeta_{1} \delta-\varepsilon_{1} \zeta_{1}\right\}\|\nabla u\|_{2}^{4} \\
& +\alpha(t)\left\{\varepsilon_{2} \delta \frac{\sigma E(0)}{l}-\frac{\sigma}{4 \alpha(0)}\right\}\left(\frac{1}{2} \frac{d}{d t}\|\nabla u\|_{2}^{2}\right)^{2} \\
& \left.+\alpha(t)\left\{\varepsilon_{1} \frac{\alpha(t)}{4}+\varepsilon_{2}\left(c(\delta)+\left(2 \delta+\frac{1}{4 \delta}\right)\right) c \alpha(t)\right)-\varepsilon_{2} \frac{\alpha^{\prime}(t) C_{3}}{\alpha(t)}\right\}(h \circ \nabla u)(t) \\
& +\alpha(t)\left\{\frac{1}{2}-\varepsilon_{2}\left(\frac{h(0) c_{p}^{2}}{4 \delta_{1}}+c\left(\delta_{1}\right)\right)\right\}\left(h^{\prime} \circ \nabla u\right)(t) \\
& +\alpha(t)\left\{\varepsilon_{1} c(\varepsilon)+\varepsilon_{2} c(\delta)+\varepsilon_{3} \beta_{1}-\frac{\eta_{0}}{\alpha(0)}\right\}\left\|u_{t}\right\|_{m}^{m} \\
& +\alpha(t)\left\{\varepsilon_{1} c(\varepsilon)+\varepsilon_{2} c(\delta)-\eta_{1} \varepsilon_{3}\right\} \int_{\tau_{1}}^{\tau_{2}}\left|\beta_{2}(s)\right|\|y(x, 1, s, t)\|_{m}^{m} d s \\
& +\alpha(t) \varepsilon_{3}\left\{-\eta_{1}-\frac{\alpha^{\prime}(t)}{\alpha(t)}\right\} \int_{0}^{1} \int_{\tau_{1}}^{\tau_{2}} s\left|\beta_{2}(s)\right| \cdot\|y(x, \rho, s, t)\|_{m}^{m} d s d \rho .
\end{aligned}
$$

Next, we carefully choose our constants.

Choose $\delta, \delta_{1}$, and $\varepsilon$ small enough such that

$$
h_{0}-\delta_{1}\left(1-c(E(0))^{p}\right)>0, \quad \delta<\frac{h_{0}}{4}, \quad \frac{\delta}{\left(l-\varepsilon\left(c_{1}+c_{2}\right)\right)}\left(\zeta_{0}+2 h_{0}^{2}\right) \alpha(0)<\frac{1}{4} h_{0} .
$$


For any fixed $\delta, \delta_{1}, \varepsilon$, we select $\varepsilon_{1}, \varepsilon_{2}$, and $\varepsilon_{3}$ so small satisfying

$$
\frac{h_{0}}{4} \varepsilon_{2}<\varepsilon_{1}<\frac{h_{0}}{2} \varepsilon_{2}
$$

and

$$
\begin{aligned}
& \varepsilon_{2} h_{0}-\varepsilon_{1}>0, \\
& \varepsilon_{2}\left[h_{0}-\delta_{1}\left(1-c(E(0))^{p}\right)\right]-\varepsilon_{1}>0 .
\end{aligned}
$$

Then, we select $\varepsilon_{1}, \varepsilon_{2}$, and $\varepsilon_{3}$ so small that (3.20) and (3.27) remain valid, and further

$$
\begin{aligned}
& \zeta_{1}\left(\varepsilon_{1}-\varepsilon_{2} \delta\right)>0, \quad \frac{\sigma}{4 \alpha(0)}-\varepsilon_{2} \delta \frac{\sigma E(0)}{l}>0, \quad \frac{1}{2}-\varepsilon_{2}\left(\frac{h(0) c_{p}^{2}}{4 \delta}+c\left(\delta_{1}\right)\right)>0, \\
& \frac{\eta_{0}}{\alpha(0)}-\varepsilon_{1} c(\varepsilon)-\varepsilon_{2} c(\delta)-\varepsilon_{3} \beta_{1}>0, \quad \eta_{1} \varepsilon_{3}-\varepsilon_{1} c(\varepsilon)-\varepsilon_{2} c(\delta)>0,
\end{aligned}
$$

where $\eta_{0}=\beta_{1}-\int_{\tau_{1}}^{\tau_{2}}\left|\beta_{2}(s)\right| d s>0$.

Therefore, (3.27) becomes, for positive constants $k_{i}, i=1, \ldots, 6$,

$$
\begin{aligned}
\mathcal{G}^{\prime}(t) \leq & -\alpha(t)\left(k_{1}+\varepsilon_{1} \frac{\alpha^{\prime}(t)}{\alpha(t)} C_{1}\right)\left\|u_{t}\right\|_{p+2}^{p+2}-\alpha(t) k_{2}\|\nabla u\|_{2}^{4} \\
& -\alpha(t)\left(k_{3}+\frac{\alpha^{\prime}(t)}{\alpha(t)}\left(\int_{0}^{t} h(\varrho) d \varrho\right)+\varepsilon_{1} \frac{\alpha^{\prime}(t) c(\kappa)}{\alpha(t)}\right)\|\nabla u\|_{2}^{2} \\
& -\alpha(t)\left(k_{4}+\frac{\alpha^{\prime}(t)}{2 \alpha(t)}\left(\varepsilon_{1}+\varepsilon_{2} C_{2}\right)\right)\left\|\nabla u_{t}\right\|_{2}^{2} \\
& +\alpha(t)\left(k_{5}-\varepsilon_{2} \frac{C_{3} \alpha^{\prime}(t)}{\alpha(t)}\right)(h \circ \nabla u)(t) \\
& -\alpha(t)\left(k_{6}+\varepsilon_{3} \frac{\alpha^{\prime}(t)}{\alpha(t)}\right) \int_{0}^{1} \int_{\tau_{1}}^{\tau_{2}} s\left|\beta_{2}(s)\right| \cdot\|y(x, \rho, s, t)\|_{m}^{m} d s d \rho .
\end{aligned}
$$

According to (2.2), $\lim _{t \rightarrow \infty} \frac{\alpha^{\prime}(t)}{\alpha(t)}=0$, we can choose $t_{1}>t_{0}$ so that (3.28) can be written as

$$
\begin{aligned}
\mathcal{G}^{\prime}(t) \leq & -\alpha(t)\left(k_{1}\left\|u_{t}\right\|_{p+2}^{p+2}+k_{2}\|\nabla u\|_{2}^{4}+k_{3}\|\nabla u\|_{2}^{2}+k_{4}\left\|\nabla u_{t}\right\|_{2}^{2}-k_{5}(h \circ \nabla u)(t)\right. \\
& \left.+k_{6} \int_{0}^{1} \int_{\tau_{1}}^{\tau_{2}} s\left|\beta_{2}(s)\right| \cdot\|y(x, \rho, s, t)\|_{m}^{m} d s d \rho\right) \\
\leq & -\alpha(t) k_{7} E(t)+\alpha(t) k_{8}(h \circ \nabla u)(t), \quad \forall t \geq t_{1} .
\end{aligned}
$$

Theorem 3.6 Suppose that (2.1)-(2.3) for any $\left(u_{0}, u_{1}, f_{0}\right)$ satisfy $E(0)>0$. Then the energy $E(t)$ of (2.5) decays to zero exponentially. That is, $\exists \lambda_{1}, \lambda_{2}>0$ such that

$$
E(t) \leq \lambda_{1} e^{-\lambda_{2} \int_{t_{1}}^{t} \alpha(\varrho) \vartheta(\varrho) d \varrho}, \quad \forall t \geq t_{1} .
$$

Proof Multiplying (3.24) by $\vartheta(t)$, using (2.1) and (2.7), we find

$$
\vartheta(t) \mathcal{G}^{\prime}(t) \leq-k_{7} \vartheta(t) \alpha(t) E(t)+k_{8} \alpha(t) \vartheta(t)(h \circ \nabla u)(t)
$$




$$
\begin{aligned}
& \leq-k_{7} \vartheta(t) \alpha(t) E(t)-k_{8} \alpha(t)\left(h^{\prime} \circ \nabla u\right)(t) \\
& \leq-k_{7} \vartheta(t) \alpha(t) E(t)-k_{8}\left\{2 E^{\prime}(t)-\alpha^{\prime}(t)\left(\int_{0}^{t} h(\varrho) d \varrho\right)\|\nabla u(t)\|_{2}^{2}\right\}
\end{aligned}
$$

Since $\vartheta(t)$ is a nonincreasing function, we have

$$
\frac{d}{d t}\left(\vartheta(t) \mathcal{G}(t)+2 k_{8} E(t)\right) \leq-k_{7} \vartheta(t) \alpha(t) E(t)-k_{8} \alpha^{\prime}(t)\left(\int_{0}^{t} h(\varrho) d \varrho\right)\|\nabla u(t)\|_{2}^{2} .
$$

From (2.6) and (2.2) that $l\|\nabla u(t)\|_{2}^{2} \leq E(t)$, we find

$$
\begin{aligned}
\frac{d}{d t}\left(\vartheta(t) \mathcal{G}(t)+2 k_{8} E(t)\right) & \leq-k_{7} \alpha(t) \vartheta(t) E(t)-k_{8} \alpha^{\prime}(t)\left(\int_{0}^{t} h(\varrho) d \varrho\right)\|\nabla u(t)\|_{2}^{2} \\
& \leq-k_{7} \alpha(t) \vartheta(t) E(t)-\frac{2 k_{8} \alpha^{\prime}(t)}{l} E(t) \\
& \leq-\alpha(t) \vartheta(t)\left(k_{7}+\frac{2 k_{8} l_{0} \alpha^{\prime}(t)}{l \vartheta(t) \alpha(t)}\right) E(t) .
\end{aligned}
$$

Since $\lim _{t \rightarrow \infty} \frac{\alpha^{\prime}(t)}{\vartheta(t) \alpha(t)}=0$, we can choose $t_{1} \geq t_{0}$ such that $k_{7}+\frac{2 k_{8} l_{0} \alpha^{\prime}(t)}{l \alpha(t) \vartheta(t)}>0$ for $t \geq t_{1}$.

Finally, let

$$
\mathcal{R}(t):=\mathcal{G}(t) \vartheta(t)+2 k_{8} E(t) \sim E(t)
$$

Hence, for some $\lambda_{2}>0$, we find

$$
\mathcal{R}^{\prime}(t) \leq-\lambda_{2} \alpha(t) \vartheta(t) \mathcal{R}(t), \quad \forall t \geq t_{1}
$$

Integrating of (3.35) over $\left(t_{1}, t\right)$ gives

$$
\mathcal{R}(t) \leq \mathcal{R}\left(t_{1}\right) e^{-\lambda_{2} \int_{t_{1}}^{t} \alpha(\varrho) \vartheta(\varrho) d \varrho}, \quad \forall t \geq t_{1} .
$$

Hence, (3.30) is established by virtue of (3.34) and (3.36). The proof is complete.

\section{Acknowledgements}

The authors would like to thank the handling editor and the referees for their relevant remarks and corrections in order to improve the final version.

Funding

Not applicable.

Availability of data and materials

Not applicable.

\section{Ethics approval and consent to participate}

Not applicable.

\section{Competing interests}

The authors declare that they have no competing interests.

Consent for publication

Not applicable.

Authors' contributions

The authors contributed equally in this article. They have all read and approved the final manuscript. 


\section{Author details}

${ }^{1}$ Department of Mathematics, Faculty of Exact Sciences, University of El Oued, El Oued, Algeria. ${ }^{2}$ Department of Matter Sciences, College of Sciences, Amar Teleji Laghouat University, Laghouat, Algeria. ${ }^{3}$ Department of Mathematics, College of Sciences and Arts, ArRass, Qassim University, Buraidah, Saudi Arabia. ${ }^{4}$ Laboratory of Fundamental and Applied Mathematics of Oran (LMFAO), University of Oran 1, Oran, 31000 Oran, Algeria.

\section{Publisher's Note}

Springer Nature remains neutral with regard to jurisdictional claims in published maps and institutional affiliations.

\section{Received: 17 July 2021 Accepted: 26 August 2021 Published online: 15 September 2021}

\section{References}

1. Adams, R., Fourier, J.: Sobolev Space. Academic Press, New York (2003)

2. Balakrishnan, A.V., Taylor, L.W.: Distributed parameter nonlinear damping models for flight structures. In: Proceedings (Damping 89), Flight Dynamics Lab and Air Force Wright Aeronautical Labs. WPAFB, Washington (1989)

3. Bass, R.W., Zes, D.: Spillover nonlinearity, and flexible structures. In: The Fourth NASA Workshop on Computational Control of Flexible Aerospace Systems. Washington: NASA Conference Publication 10065, pp. 1-14 (1991)

4. Bathory, M., Bulíček, M., Málek, J.: Large data existence theory for three-dimensional unsteady flows of rate-type viscoelastic fluids with stress diffusion. Adv. Nonlinear Anal. 10(1), 501-521 (2021)

5. Benbernou, S., Gala, S., Ragusa, M.A.: On the regularity criteria for the 3D magnetohydrodynamic equations via two components in terms of BMO space. Math. Methods Appl. Sci. 37(15), 2320-2325 (2014)

6. Bland, D.R.: The Theory of Linear Viscoelasticity. Courier Dover Publications, Mineola (2016)

7. Boulaaras, S., Choucha, A., Ouchenane, D., Cherif, B.: Blow up of solutions of two singular nonlinear viscoelastic equations with general source and localized frictional damping terms. Adv. Differ. Equ. 2020, 310 (2020)

8. Boulaaras, S., Draifia, A., Zennir, Kh.: General decay of nonlinear viscoelastic Kirchhoff equation with Balakrishnan-Taylor damping and logarithmic nonlinearity. Math. Methods Appl. Sci. 42, 4795-4814 (2019)

9. Cavalcanti, M., Domingos Cavalcanti, V., Lasiecka, I., Webler, C.: Intrinsic decay rates for the energy of a nonlinear viscoelastic equation modeling the vibrations of thin rods with variable density. Adv. Nonlinear Anal. 6(2), 121-145 (2017)

10. Choucha, A., Boulaaras, S., Ouchenane, D., Beloul, S.: General decay of nonlinear viscoelastic Kirchhoff equation with Balakrishnan-Taylor damping, logarithmic nonlinearity and distributed delay terms. Math. Methods Appl. Sci. 44(7), 5436-5457 (2021). https://doi.org/10.1002/mma.7121

11. Choucha, A., Boulaaras, S.M., Ouchenane, D., Cherif, B.B., Abdalla, M.: Exponential stability of swelling porous elastic with a viscoelastic damping and distributed delay term. J. Funct. Spaces 2021, Article ID 5581634 (2021). https://doi.org/10.1155/2021/5581634

12. Choucha, A., Ouchenane, D., Boulaaras, S.: Well posedness and stability result for a thermoelastic laminated Timoshenko beam with distributed delay term. Math. Methods Appl. Sci. 43(17), 9983-10004 (2020). https://doi.org/10.1002/mma.6673

13. Choucha, A., Ouchenane, D., Boulaaras, S.: Blow-up of a nonlinear viscoelastic wave equation with distributed delay combined with strong damping and source terms. J. Nonlinear Funct. Anal. 2020, Article ID 31 (2020). https://doi.org/10.23952/jnfa.2020.31

14. Choucha, A., Ouchenane, D., Zennir, Kh., Feng, B.: Global well-posedness and exponential stability results of a class of Bresse-Timoshenko-type systems with distributed delay term. Math. Methods Appl. Sci., 1-26 (2020). https://doi.org/10.1002/mma.6437

15. Coleman, B.D., Noll, W.: Foundations of linear viscoelasticity. Rev. Mod. Phys. 33(2), 239 (1961)

16. Feng, B., Soufyane, A.: Existence and decay rates for a coupled Balakrishnan-Taylor viscoelastic system with dynamic boundary conditions. Math. Models Methods Appl. Sci. 43, 3375-3391 (2020)

17. Gala, S., Galakhov, E., Ragusa, M.A., Salieva, O.: Beale-Kato-Majda regularity criterion of smooth solutions for the Hall-MHD equations with zero viscosity. Bull. Braz. Math. Soc. (2021). https://doi.org/10.1007/s00574-021-00256-7

18. Gheraibia, B., Boumaza, N.: General decay result of solution for viscoelastic wave equation with Balakrishnan-Taylor damping and a delay term. Z. Angew. Math. Phys. 71, 198 (2020). https://doi.org/10.1007/s00033-020-01426-1

19. Kirchhoff, G.: Vorlesungen uber Mechanik. Tauber, Leipzig (1883)

20. Liu, W., Zhu, B., Li, G., Wang, D.: General decay for a viscoelastic Kirchhoff equation with Balakrishnan-Taylor damping, dynamic boundary conditions and a time-varying delay term. Evol. Equ. Control Theory 6, 239-260 (2017)

21. Makvand Chaharlang, M.M., Razani, A.: Two weak solutions for some Kirchhoff-type problem with Neumann boundary condition. Georgian Math. J. 28(3), 429-438 (2021)

22. Mesloub, F., Boulaaras, S.: General decay for a viscoelastic problem with not necessarily decreasing kernel. J. Appl. Math. Comput. 58, 647-665 (2018). https://doi.org/10.1007/S12190-017-1161-9

23. Mezouar, N., Boulaaras, S.: Global existence and decay of solutions for a class of viscoelastic Kirchhoff equation. Bull. Malays. Math. Sci. Soc. 43, 725-755 (2020)

24. Mezouar, N., Boulaaras, S.: Global existence and exponential decay of solutions for generalized coupled non-degenerate Kirchhoff system with a time varying delay term. Bound. Value Probl. (2020) https://doi.org/10.1186/s13661-020-01390-9

25. Mingqi, X., Radulescu, V.D., Zhang, B.: Nonlocal Kirchhoff diffusion problems: local existence and blow-up of solutions. Nonlinearity 31(7), 3228-3250 (2018)

26. Mingqi, X., Radulescu, V.D., Zhang, B.: Nonlocal Kirchhoff problems with singular exponential nonlinearity. Appl. Math Optim. 84(1), 915-954 (2021)

27. Mu, C., Ma, J.: On a system of nonlinear wave equations with Balakrishnan-Taylor damping. Z. Angew. Math. Phys. 65 91-113 (2014)

28. Nicaise, A.S., Pignotti, C.: Stabilization of the wave equation with boundary or internal distributed delay. Differ. Integral Equ. 21(9-10), 935-958 (2008) 
29. Ouchenane, D., Boulaaras, S., Mesloub, F.: General decay for a viscoelastic problem with not necessarily decreasing kernel. Appl. Anal. 98(44), 1-17 (2018). https://doi.org/10.1080/00036811.2018.1437421

30. Polidoro, S., Ragusa, M.A.: Sobolev-Morrey spaces related to an ultraparabolic equation. Manuscr. Math. 96(3), 371-392 (1998)

31. Razani, A.: Shock waves in gas dynamics. Surv. Math. Appl., 2, 59-89 (2007)

32. Razani, A.: Subsonic detonation waves in porous media. Phys. Scr. 94, 085209, 6 pages (2019). https://doi.org/10.1088/1402-4896/ab029b

33. Tian, L., Cheng, Z:: A triangular plate bending element based on discrete Kirchhoff theory with simple explicit expression. Mathematics 9(11), 1181 (2021)

34. Wu, Y., Qiao, Z.H., Hamdani, M.K., Kou, B.Y., Yang, L.B.: A lass of variable-order fractional p(.)-Kirchhoff-type systems. J. Funct. Spaces 2021, 5558074 (2021)

35. Zarai, A., Tatar, N.: Global existence and polynomial decay for a problem with Balakrishnan-Taylor damping. Arch. Math. 46, 157-176 (2010)

Submit your manuscript to a SpringerOpen ${ }^{\circ}$ journal and benefit from:

- Convenient online submission

- Rigorous peer review

Open access: articles freely available online

- High visibility within the field

- Retaining the copyright to your article

Submit your next manuscript at $\gg$ springeropen.com 